\section{Virtual Pinhole Microscope \\ A Revolution in Confocal Microscopy}

Los Alamos Laboratory and VayTek, Inc. recently announced the development of a prototype for the "Scanning Computed Confocal Imager" or Virtual Pinhole Microscope. This breakthrough technology takes advantage of existing improvements in digital image systems, but configures functional subsystems in a novel way that eliminates many of the limitations and much of the expense associated with conventional confocal imagery.

One of the key elements of the new Imager is the use of a "virtual" aperture. The virtual aperture is implemented in software on a host computer, such as a pentium-based computer or a Power Mac. It's possible, with this system, to adjust key parameters (such as effective aperture size) to optimize the image even after the basic data are acquired. Several algorithms have been used to produce images similar to those acquired through conventional confocal microscopy.

A key subsystem of the Virtual Pinhole Microscope offers an elegant solution for providing scanned illumination. John George, PhD in Biophysics and Neuroscience at Los Alamos, uses spatial light modulators (SLMs) to produce time-varying spatial patterns of illumination. The SLM that $\mathrm{Dr}$. George is using on the current prototype is a liquid crystal display (LCDs) similar to those used in some video projectors. By setting the polarization on the LCDs, Dr. George can program light patterns from a single point to a complicated spatial pattern, to one or more lines or slits - choosing optimal patterns in space and time for capturing the desired images. Other technologies, such as ferroelectric liquid crystals, micro-mirror display systems, or electronic laser scanning systems can be adapted for use with the Scanning Computed Confocal Imager

Other Features of the Virtual Pinhole Microscope Include:

- No Moving Parts - provides an excellent basis for a confocal and spectroscopic endoscope system.

- Easy to Retrofit - adaptable to existing systems, including conventional microscopes with video capability.

- Low Cost - high performance, extended sensitivity, speed, and dynamic range from low cost components.

- Can be configured to operate in transmitted light modes, reflected light, or epifluorescence modes.

- Multiple images with different effective contrast mechanisms can be computed from a single data set.

- All attributes of the system are programmable.

Examples of Applications for the Virtual Pinhole Microscope Include:

- Optical Imaging of Neural Function - Maximizing the ability to obtain confocal images in real time, researchers could measure changes in the optical properties of the cortex that reflect neuronal activity. Virtual Pinhole Confocal techniques would allow different response properties at different cortical layers to be measured at a much higher resolution. These

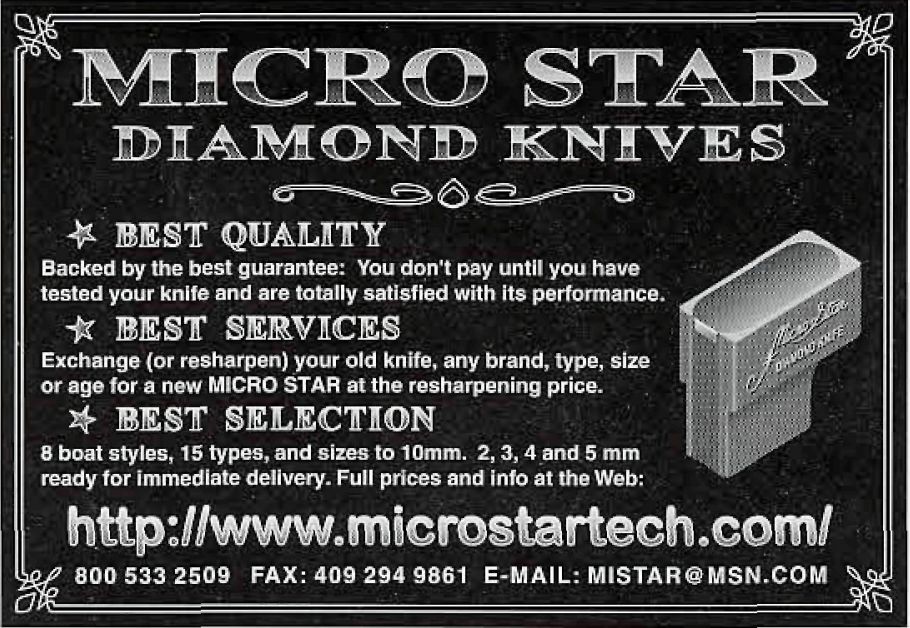

measurements could describe the spatial response pattern across the cortex at each of the cortical layers and sublayers.

- High Speed Imaging - Although most of the power in macroscopic electrophysiological measurements of neural population activity is at frequencies between 10 and $100 \mathrm{~Hz}$, firing rates of individual neurons may approach $1 \mathrm{kHz}$. Oscillatory population responses are observed in a range of $40-60 \mathrm{~Hz}$. It would be useful to resolve phase relationships between active neural populations. Existing technical approaches using slow scan or standard video devices cannot address important temporal dynamic regimes within single cells or networks of cells.

- Engineering and Material Science - The new system offers a rugged, lighter weight imager with great flexibility and improved reliability.

- Inspection and Industrial Quality Control - Most inspection sites, such as those associated with semi-conductor inspection, have invested heavily in expensive equipment. The case with which the Virtual Pinhole

Microscope can be retrofit to existing systems makes it ideal for these systems.

A complete Virtual Pinhole Microscope system consists of imaging optics (an existing microscope, for example), a system for scanned illumination, a standard or high performance solid state video camera, and a computer system for image acquisition, scan control, and image reconstruction.

Dr. John George and his colleagues at Los Alamos National Laboratory have a patent pending on the system. VayTek, Inc. has contributed expertise in digital imaging techniques and has licensed the Virtual Pinhole Microscope for manufacture and distribution.

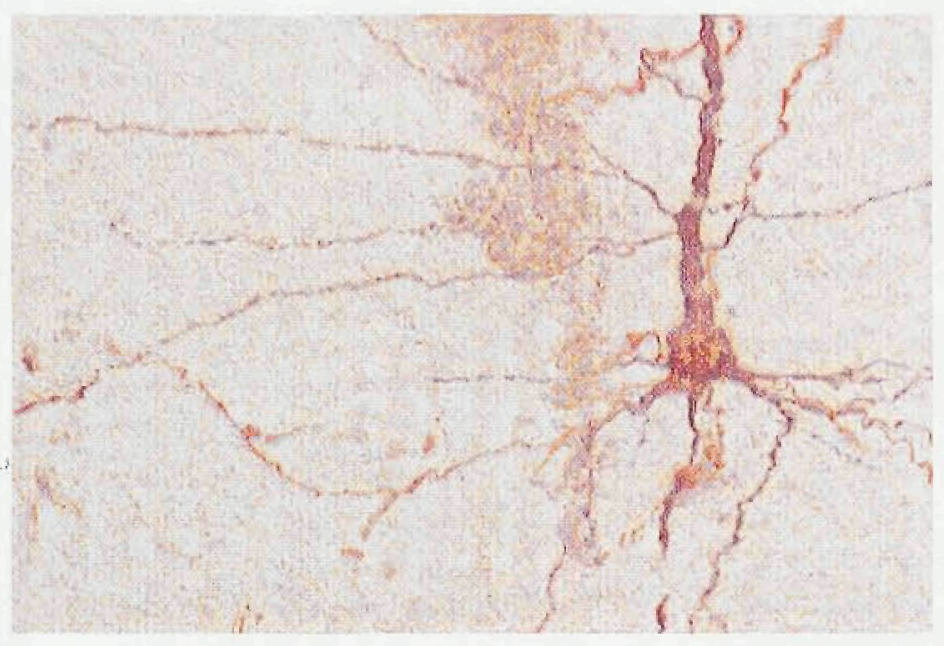

Rat brain nerve cell reconstructed from a series of confocal slice images collected with Virtual Pinhole Microscope system

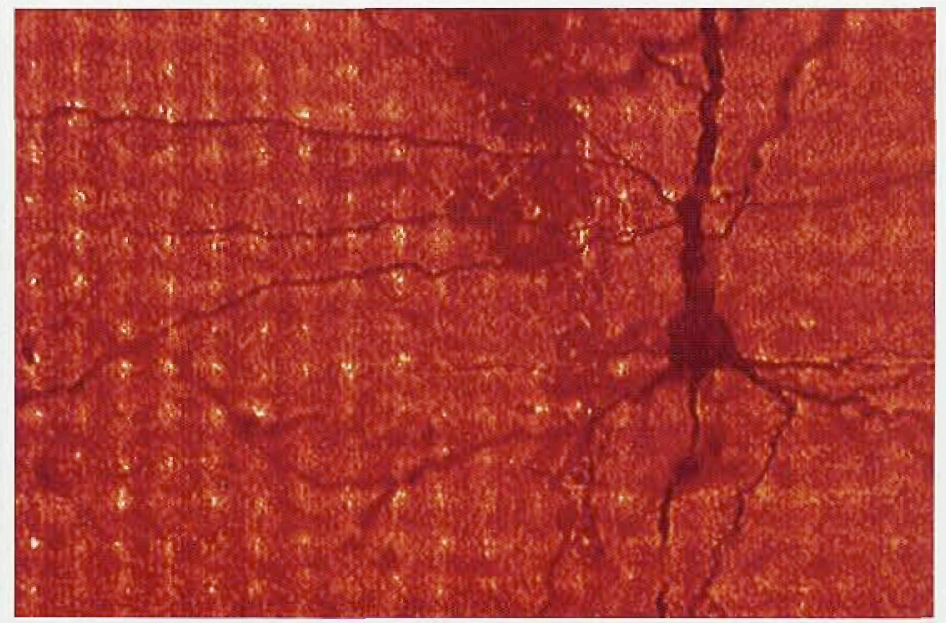

LCD illumination grid evident in image acquired with Virtual Pinhole Microscope. One of a series of confocal slices reconstructed later to create a 3D image of a rat brain nerve cell 


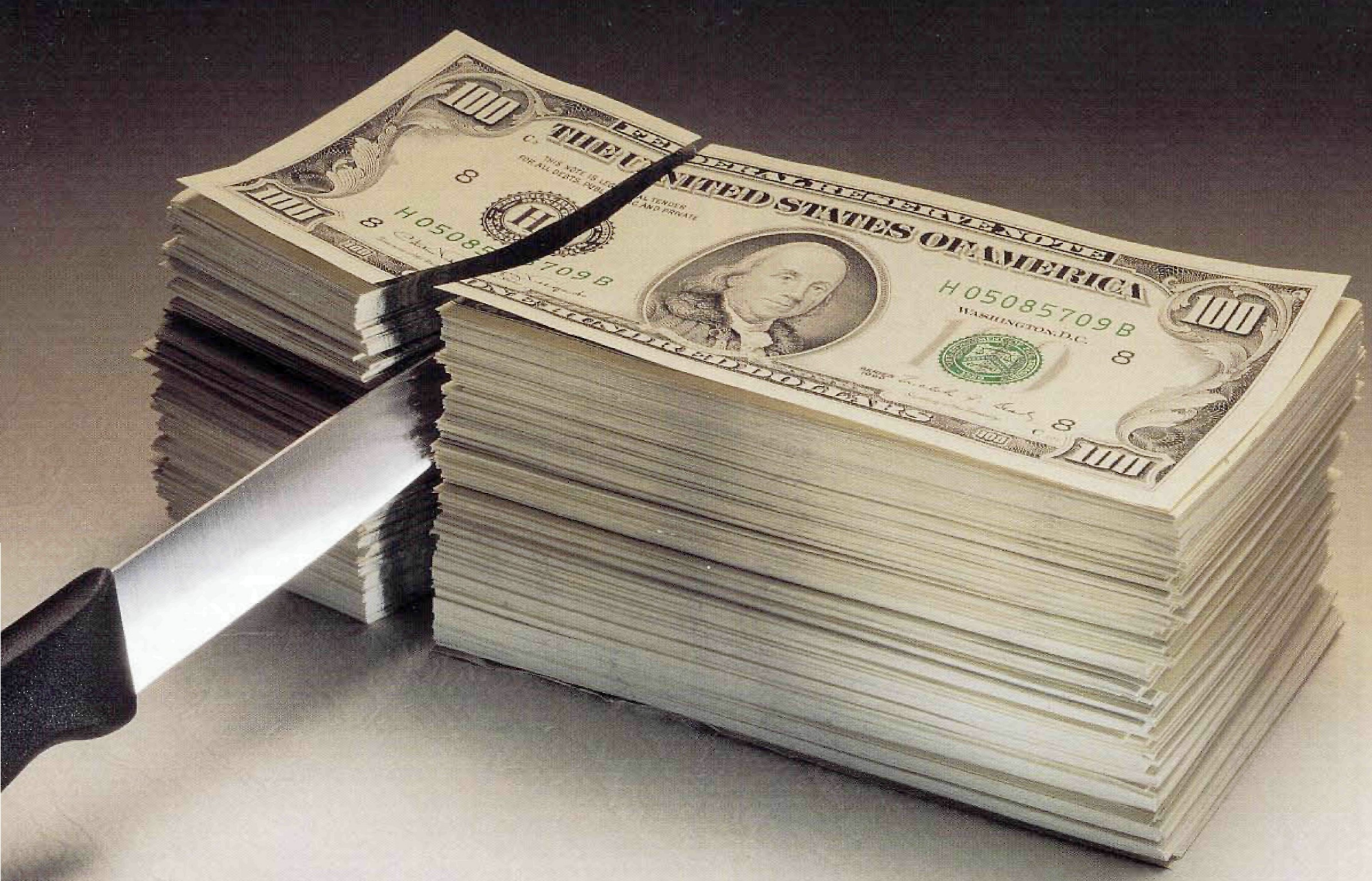

\section{It's Every Bit True Field Emission... Except The Cost.}

Introducing Topcon

SM-520 Field Emission Scanning Electron Microscope

At last, you can enjoy the benefits of Field Emission performance on a limited budget. With the SM-520, you get the high resolution, reliability, versatility, and low $\mathrm{kV}$ performance you want. Plus Topcon's unique Dual Control system that's easy to use and adapts to your personal operating style.
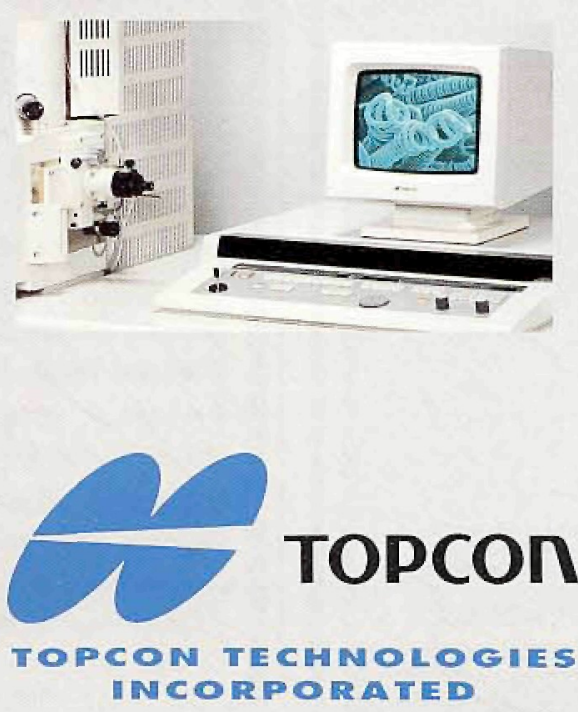

Field Emission Performance at $\mathrm{LaB}_{6}$ Prices

The best part is, it all comes at $\mathrm{LaB}_{6}$ prices. Take a closer look and you'll see that the SM-520 is within your reach. Call us today at 1-800-538-6850 or write to Topcon Technologies, Inc., 37 West Century Road, Paramus, New Jersey 07652. 